\title{
THE HIP
}

\author{
P Sanville, D A Nicholson, P A Driscoll
}

The hip joint is a common site for trauma in adults and damage to the hip may be associated with injuries to the rest of the pelvis, the femur, and the knee. This chapter describes a system of assessment to help interpret hip radiographs.

\section{Important anatomical considerations}
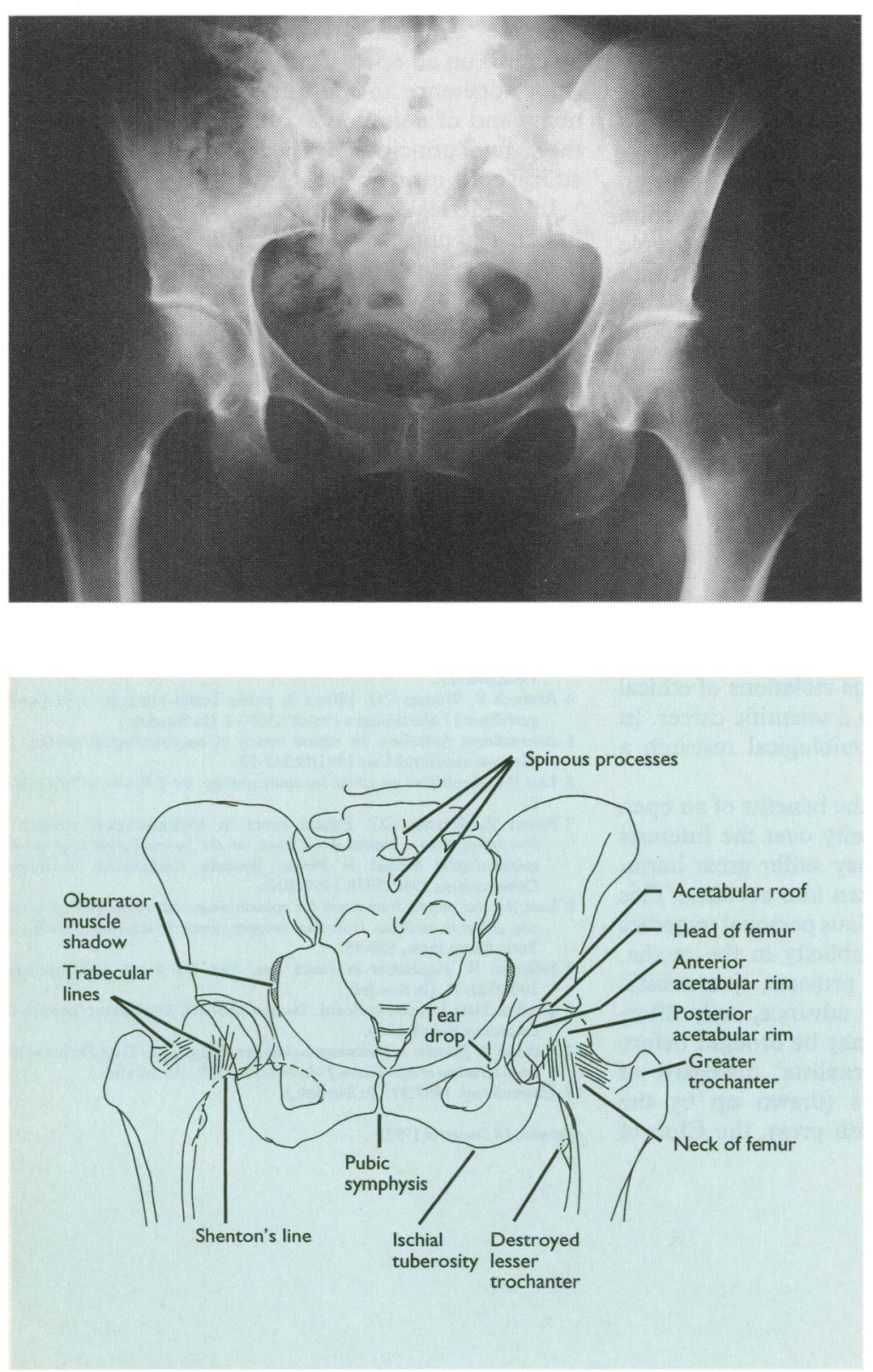

FIG 1-Standard anteroposterior view and line diagram of the hips. Note that the left lesser trochanter is destroyed by a metastasis.

\section{Adult}

The strong hip joint capsule and the surrounding large muscle bulk prevent dislocation except in severe trauma. More commonly the hip is fractured with a resulting high incidence of avascular necrosis of the femoral head, complicating intracapsular fractures, and epiphyseal injuries. The trochanteric apophyses are the insertion points for the gluteus medius (greater trochanter) and iliopsoas (lesser trochanter) muscles and are prone to avulsion in athletic adolescents. In malignant disease the proximal femur, particularly the subtrochanteric region, is a common site for metastases and pathological fracture (fig 2).

\section{Children}

The proximal capital femoral epiphysis is present from the age of 3 months until 18-20 years, with double epiphyseal ossification centres being common. Although asymmetry, irregular contour, and notching of the epiphyses can be normal variants in young children, a smaller epiphysis or any asymmetry in children with symptomatic hips may indicate injury. Flattening of the epiphysis is abnormal. 


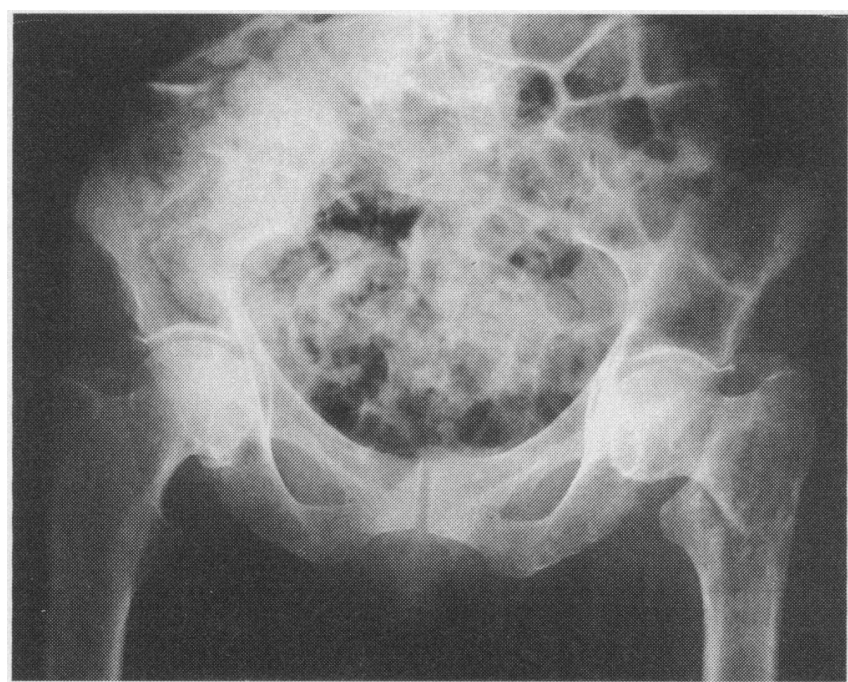

The metaphysis has a rich blood supply and is a common site for osteomyelitis and consequently septic arthritis.

FIG 2-Left intertrochanteric pathological fracture. Note the large lucent trochanteric metastasis and disruption of Shenton's line.

\section{Mechanisms of injury}

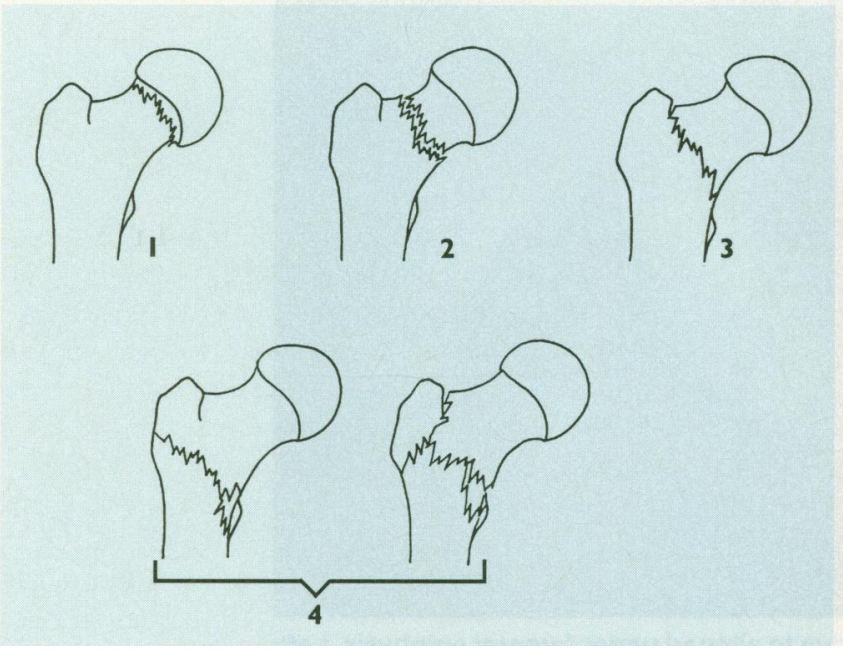

FIG 3-Sites of fracture of the femoral neck: (1) subcapitalintracapsular (most common site); (2) transcervicalintracapsular; (3) intertrochanteric-extracapsular fracture line along base of neck; (4) pertrochanteric-extracapsular without and with extension into proximal shaft as a spiral fracture.

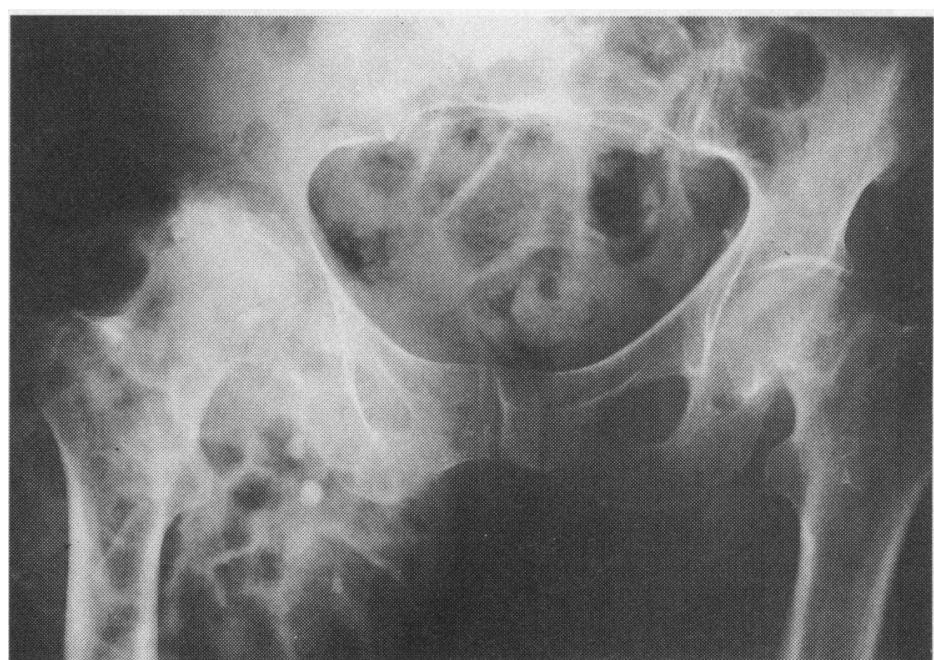

FIG 4-Left subcapital fracture. Note the upward displacement of the femur and abnormal Shenton's line. There is severe osteoarthritis of the right hip and a massive right inguinal hernia containing small bowel loops.

\section{Fractured neck of femur in children}

Delbet classification:

Type 1-Transepiphyseal (avascular necrosis usually follows)

Type 2-Transcervical (avascular necrosis common if displaced)

Type 3-Cervicotrochanteric

Type 4-Pertrochanteric
Femoral neck fractures

These fractures are seen most commonly in elderly osteopenic women after a fall. They also occur in men under 60 years after industrial accidents.

There are four sites of fracture (fig 3). Fractures may be intracapsular or extracapsular and are usually visible in the anteroposterior film as a lucent line. Pertrochanteric fractures are commonly comminuted with displacement of the lesser trochanter.

In children considerable violence is needed to fracture the neck of the femur. In transepiphyseal fractures the capital epiphysis is separated from the metaphysis and dislocated out of the acetabulum, often resulting in avascular necrosis. 


\section{Complications of hip dislocation}

Slipped femoral epiphysis

Sciatic nerve palsy

Femoral nerve or artery compression

(anterior dislocation)

Failed reduction and recurrent dislocation

Avascular necrosis of the femoral head Osteoarthritis

Myositis ossificans

\section{Dislocation}

The femoral head can be dislocated anteriorly, posteriorly, or centrally. Central dislocation occurs when the femoral head impacts through the acetabulum because of a sideways fall, a blow to the greater trochanter, or a fall from a height on to the feet. Falling on to the feet is often associated with a fracture of the anterior or posterior pelvic columns (see article on pelvis, 9 October, p 930). Posterior dislocation may result from a blow to the lumbar spine-for example, from falling masonry-with the hip flexed. In patients who have hit a car dashboard dislocation of the hip is associated with a fracture of the femoral shaft or patella.

\section{Slipped upper femoral epiphysis (adolescent coxa vara)}

In this condition the femoral neck moves proximally and externally rotates on the unfused epiphysis. It is bilateral in $20 \%$ of cases and occurs in overweight, hypogonadal, or tall thin adolescents. Pain, sometimes referred to the knee, or limp is a common presenting symptom. Both hips should be evaluated. Early slip is best assessed in the lateral film (fig 5).
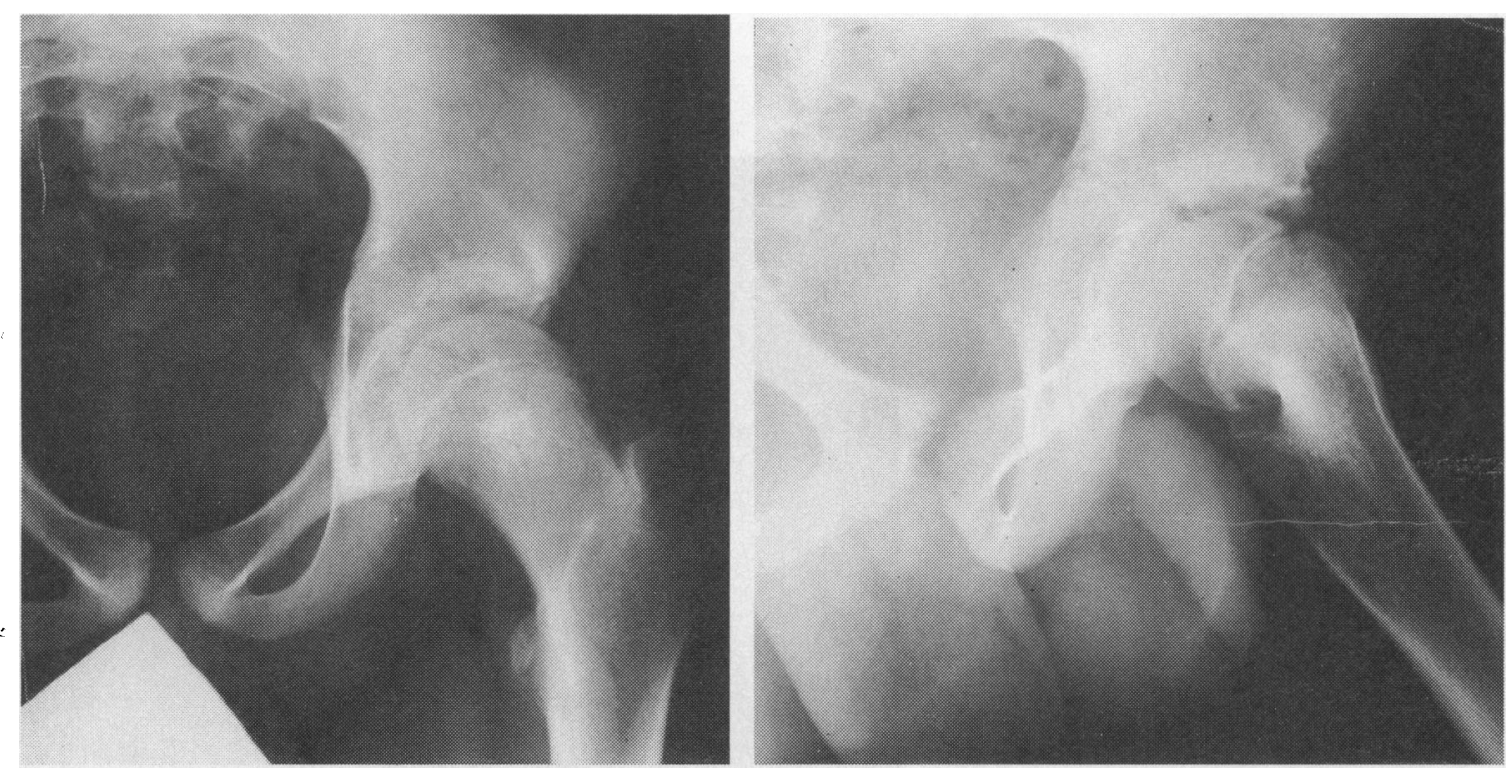

FIG 5-Radiographs of a 13 year old boy with an acutely painful left hip due to slipped upper femoral epiphysis. Left: anteroposterior coned film - the slip is not apparent. Note gonad protection. Right: oblique view of left hip. The femoral neck has slipped proximally over the epiphysis, which remains in the acetabulum, and externally rotated resulting in a prominent lesser trochanter and superimposed greater trochanter.

\section{Types of view}

\author{
Radiographic views of the hip \\ Standard: \\ Anteroposterior \\ Cross table lateral \\ Additional \\ Frog leg lateral \\ Oblique
}

Routine radiographic examination should include an anteroposterior view of the whole pelvis (fig 1), which allows comparison between both hips, and a lateral view of the symptomatic joint if an abnormality is seen in the anteroposterior view or if slipped epiphysis is suspected.

Coned oblique views can be taken to assess acetabular fractures, undisplaced femoral neck fractures, and trochanteric fractures. The frog leg lateral film is useful in children. It shows the femoral head and neck in a position between the anteroposterior and the standard lateral projection.

Anteroposterior and lateral views of the femoral shaft and knee are indicated when there is a history of severe trauma or when clinical findings suggest more than one fracture site. Gonad protection should always be used in children and adults of reproductive age, providing it will not obscure a fracture. 


\section{System of radiological assessment}

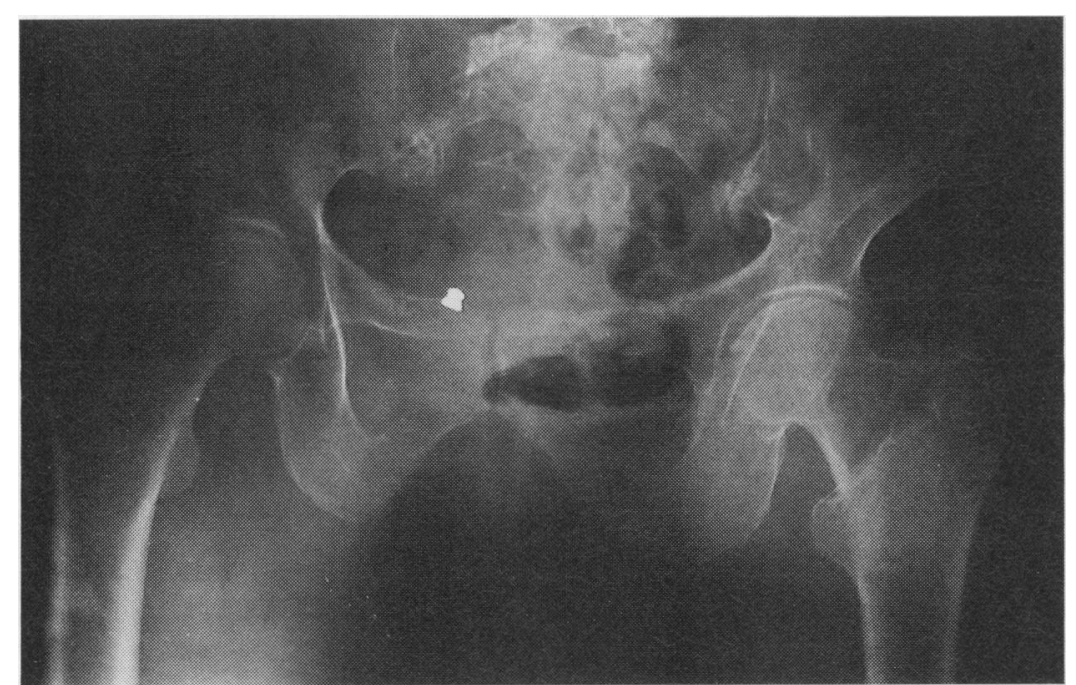

FIG 6-Undisplaced left pertrochanteric fracture. Note the lucent line extending into a break in the cortex of the greater trochanter. An air gun pellet is lying in the abdominal wall.
Follow the ABCs system of alignment, bone margin, cartilage and joints, and soft tissue.

\section{Anteroposterior projection}

Check the adequacy and quality of the radiograph-The anteroposterior film centred just above the pubic symphysis should include the whole pelvis and proximal third of the femur. The lumbar spinous processes, coccyx, and pubic symphysis will form a vertical straight line in a non-rotated film.

Check alignment of bones-The medial half of the femoral head overlaps the posterior acetabular rim. The curve of the lower border of the superior pubic ramus and the inferior aspect of the neck of the femur should form a smooth arc (Shenton's line).
The plain film usually shows no abnormality in children presenting with an irritable hip. However, orthopaedic referral is mandatory
Check bone margins and density - Trace around the margin of the proximal femur, starting at the inferior aspect of the medial femoral cortex and checking for any disruption of the cortical line, particularly around the neck or trochanters (fig 6). In patients who can bear weight, carefully examine the trabecular lines and cortical margins of the femoral neck in a coned anteroposterior film to detect undisplaced fractures. Undisplaced femoral neck fractures will have a discontinuity in the trabecular lines, with an associated linear increase in density when impacted (figs 7 and 8 ). Check the cortical lines of the acetabular joint surface and posterior and anterior rim. Both hips should be symmetrical.
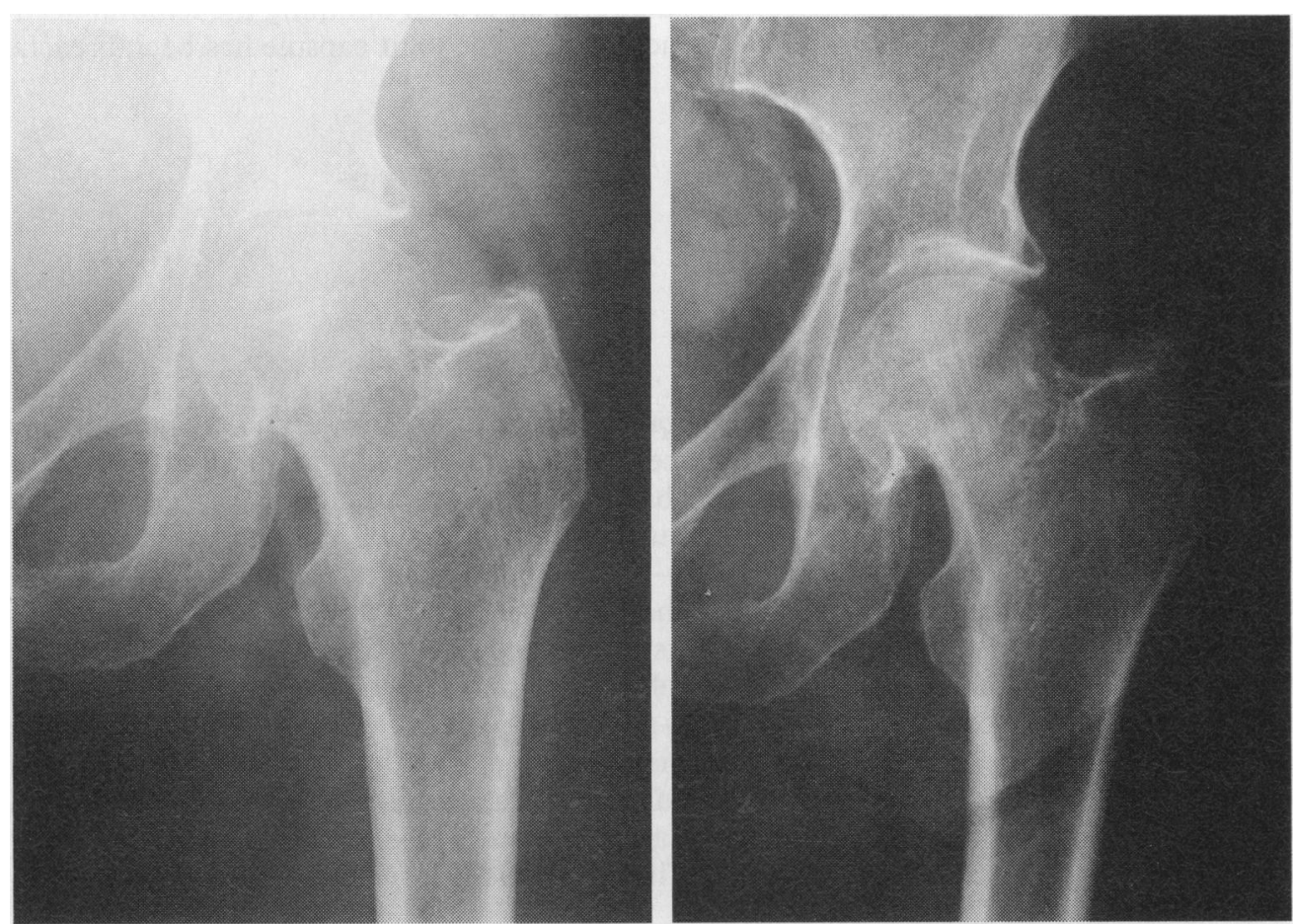

FIG 7-Left: missed undisplaced left subcapital fracture. Note the disruption of the trabecular lines. Right: same patient three weeks later. III defined areas of sclerosis are seen in the fracture line due to impaction. Shenton's line is now disrupted. 


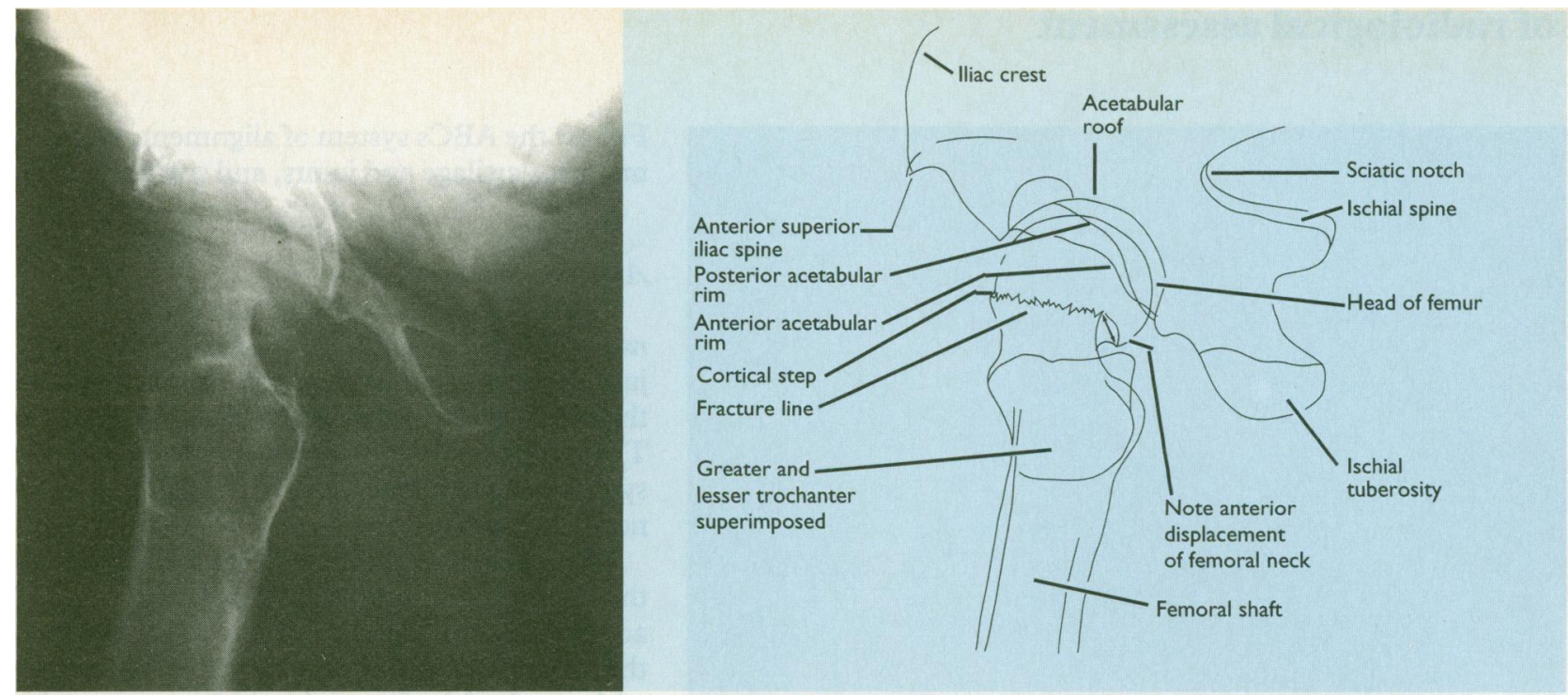

FIG 8-Cross table lateral film and line diagram of the left hip (of the same patient as in figure 7). The femoral neck is displaced anteriorly and impacted into the head of femur.

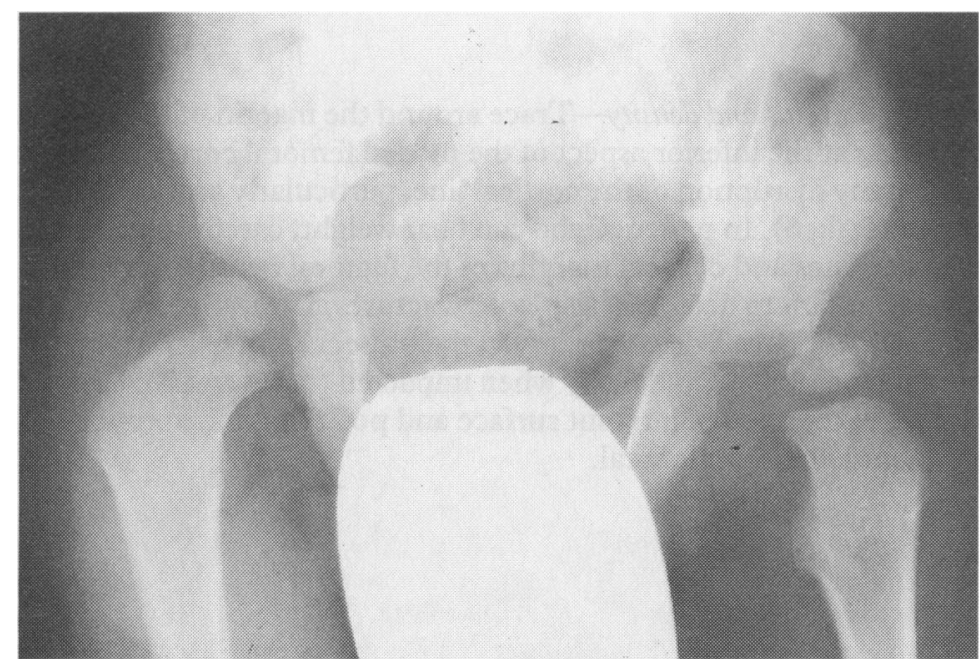

FIG 2 -Acute septic arthritis of the left hip in a 1 year old boy. The left femoral capital epiphysis is greatly displaced out of the acetabulum by an effusion and is starting to disintegrate. Emergency drainage is required.
Cartilage and joints - In children widening of the joint space between the tear drop and the cortex of the femoral head may be seen in joint effusions (fig 9). A difference greater than $2 \mathrm{~mm}$ between the two sides is clinically important. Check that the physis (growth plate) is not widened or compressed (Salter-Harris types 1 and 5 fractures).

Check soft tissues-Because of the large muscle mass around the hip, soft tissue injuries are not visible in plain radiographs. Air or a metallic foreign body will be visible after a penetrating injury. If air is seen outlining the femoral head or acetabulum the joint capsule has been breached.

\section{Acetabular fractures}

- Are easily missed in the anteroposterior film and can simulate normal variants

- Can be associated with an isolated superior pubic ramus fracture

- May be complicated by sciatic nerve palsy and severe intrapelvic haemorrhage

\section{The lateral projection}

Check the adequacy and quality of the radiograph.-The cross table lateral film should include the acetabulum, the ischial spine and tuberosity, and the proximal femur. The trochanters overlap. In the frog leg lateral film, the greater trochanter should be projected over the neck of femur.

Check alignment of bones-The femoral neck lies anteverted about $30^{\circ}$ to the femoral shaft. Check that the entire metaphysis is covered by epiphysis in children and adolescents. In slipped upper femoral epiphysis the centre of the femoral neck metaphysis lies anterior to its normal position over the central epiphysis (figs 4 and 5). In patients with dislocated hips the cross table lateral film will define whether dislocation is anterior or posterior.

Check bone margins and density - Trace around the margins of the femur and then the acetabulum and ischium. If a dislocation is present look for acetabular fragments. These are usually displaced in the same direction as the femoral head.

Check soft tissues 